\title{
MAHOU Y CORONITA: ANALISIS COMPARADO DEL VALOR DE MARCA PARA EL CONSUMIDOR ESPAÑOL
}

\author{
Cristina Calvo Porral ${ }^{1}$ \\ Paulino Montes Solla ${ }^{2}$ \\ Universidad de La Coruña \\ ccalvo@udc.es
}

Material original autorizado para su primera publicación en la revista

académica REDMARKA. Revista Digital de Marketing Aplicado.

http://doi.org/10.17979/redma.2012.02.09.4752

Recibido: 16 Octubre 2012

Aceptado 13 Diciembre 2012

\section{RESUMEN}

Introducción: El sector cervecero es un sector económico y de actividad fundamental dentro del panorama agroalimentario español, sin embargo existen pocos estudios que analicen el valor de marca de este producto.

Objetivos: El presente estudio plantea una aproximación al perfil del consumidor de cerveza español y un análisis del valor de marca de una marca nacional y otra marca importada.

Metodología: Se lleva a cabo un estudio cuantitativo a consumidores y se realiza un análisis de las variables del valor de marca, para comparar dos marcas presentes en el mercado español -Mahou y Coronita-.

\footnotetext{
${ }^{1}$ Cristina Calvo es Licenciada en Administración y Dirección de Empresas por el ICADE de Madrid (Universidad Pontificia de Comillas), y también es Licenciada en Investigación y Técnicas de Mercado por el ICADE. Es Doctora en Economía y cuenta con diversas publicaciones en el área de marketing. Su carrera profesional se ha desarrollado durante más de diez años en el sector textil, como técnico de comercio exterior en el área de Expansión Internacional de Carolina Herrera y Purificación García (Sociedad Textil Lonia). Actualmente es profesora del Departamento de Análisis Económico y Administración de Empresas, de Facultad de Economía y Empresa de Universidad de La Coruña.

${ }^{2}$ Paulino Montes es Licenciado en Ciencias Económicas y Empresariales por la Universidad de La Coruña. Colabora desde hace más de siete años en el Grupo Jean Monnet de Competencia y Desarrollo en la Unión Europea. Actualmente es asistente de la Cátedra Jean Monnet de Economía Industrial Europea, de la Universidad de La Coruña. Es experto en temas de Desarrollo regional, y ha desempeñado durante los últimos cinco años tareas de consultoría y evaluación de los fondos FEDER de la Comisión Europea en España.
} 
Resultados: Los resultados sugieren una mejor valoración de la marca Coronita por parte de los consumidores españoles.

Conclusiones: A la vista de los resultados obtenidos, se puede afirmar que la marca Coronita ha sabido posicionarme adecuadamente en el mercado español, haciendo una oferta de valor para los consumidores.

Palabras clave: Valor de marca, notoriedad de marca, cerveza.

\section{ABSTRACT}

MAHOU AND CORONITA: COMPARATIVE ANALYSIS OF THE MARK VALUE FOR THE SPANISH CONSUMER

Introduction: The Spanish brewing industry is a main economic sector and a major activity in the agrifood area, however there are few studies regarding the analysis of Brand Equity in this product.

Objectives: This study developes an approximation to the profile of the Spanish beer consumer and the analysis of brand equity for a domestic and an imported brand.

Methodology: For this purpose, a quantitative study is carried out, proceeding with an analysis of the variables of brand equity in order to compare two brands available in the Spanish market -Mahou and Coronita-.

Results: Results suggest a better evaluation of the beer brand Coronita by the Spanish consumers.

Conclusions: Attending the results obtained, we may state that Coronita brand has been positioned properly in the Spanish market, making a valuable proposition to consumers.

Keywords: Brand Equity, Brand Awareness, beer. 


\section{INTRODUCCION}

El sector cervecero es un sector económico y de actividad clave dentro del panorama agroalimentario español y la cerveza es la primera bebida generadora de empleo directo e indirecto (Ministerio de Medio ambiente y Medio Rural, 2010). Dentro de este contexto, existen escasos estudios sobre el valor de marca en este sector, desde el punto de vista del consumidor (Atilgan et al., 2005). El presente trabajo trata de contribuir a la carencia en esta área, aplicando el modelo propuesto por Aaker a un sector concreto, como es el de la cerveza y más concretamente se centra en el mercado español, con el objetivo de analizar el valor de marca de un producto con gran demanda y popularidad, comparando para ello dos marcas -Mahou y Coronita- y analizando sus diferencias. El estudio se ha estructurado de la siguiente forma. Se comienza con la fundamentación teórica, a continuación se plantean los objetivos. En el apartado cuarto se explica la metodología empleada, las variables seleccionadas, las escalas de medida empleadas. A continuación, se comentan los resultados obtenidos, para finalizar con las conclusiones, implicaciones y limitaciones de la investigación.

\section{EL VALOR DE MARCA}

Farquhar (1989) define el valor de marca como el valor añadido que una marca determinada aporta a un producto o servicio, y señala que el valor de marca es aquel conjunto de activos y pasivos vinculados a una marca, su nombre y símbolo, que incorporan o disminuyen el valor aportado por un producto o servicio a la compañía o a sus clientes. Por otro lado, Keller (1993), define el valor de marca como el efecto diferencial del conocimiento de la marca en la respuesta dada por el consumidor al marketing de marca. De acuerdo con Aaker (1991), el valor de marca se puede definir como el conjunto de activos unidos a la marca, su nombre o logotipo, que bien añaden o reducen el valor proporcionado por un producto o servicio de una compañía a sus clientes. Existe consenso en torno a la idea de que el valor de marca constituye un elemento diferencial, generado por los signos distintivos de la marca, y que 
dicha diferenciación puede ser generada por diversas fuentes o componentes del valor de marca.

Aaker $(1991,1996)$ y Keller $(1993)$ han conceptualizado el concepto de valor de marca como una variable multidimensional y proponen modelos para la medida del valor de marca, caracterizados por el empleo de variables relacionadas con el comportamiento de los consumidores, sus percepciones y preferencias. Numerosos modelos teóricos ponen también de relieve el carácter multidimensional del valor de marca (Lassar et al., 1995; Agarwal y Rao, 1996, Kim et al., 2008), así como las variables que condicionan dicho valor (Yoo y Donthu, 2002; Pappu, et al., 2005). De entre todos estos modelos, los propuestos por Aaker $(1991,1996)$ y Keller $(1993)$, son los que han tenido una mayor aceptación en la literatura (Yoo y Donthu, 2001; Pappu et al., 2005, 2007; Jung y Sung, 2008). Por este motivo, en este estudio se ha optado por definir el valor de marca siguiendo el planteamiento de Aaker, debido a que representa un referencia importante en la comunidad científica del marketing y porque se ha demostrado empíricamente la relación existente entre las dimensiones del valor de marca recogidas por dicho modelo, y el valor de marca para el consumidor (Yoo et al., 2000). Por consiguiente, siguiendo a Aaker (1991), el valor de marca está formado por cinco dimensiones que son la notoriedad de marca, la lealtad de marca, la calidad percibida, las asociaciones de marca -o imagen de marca-, y otros activos vinculados a la marca.

En primer lugar, la notoriedad de marca es un concepto relacionado con el reconocimiento y el recuerdo que el consumidor tiene de una marca determinada, así como de su habilidad para identificar la marca en determinadas situaciones (Rossiter y Percy, 1987). El papel que desempeña la notoriedad en el valor de marca depende del nivel de notoriedad que ha alcanzado dicha marca en el mercado, de manera que cuanto mayor es su notoriedad, mayor predominio tendrá la marca, y esto incrementará la probabilidad de que la marca sea considerada en las situaciones de compra. Por lo tanto, un incremento de la notoriedad, incrementará la influencia de la marca en las decisiones de compra de los consumidores (Nedungadi, 1990). 
La segunda variable del valor de marca, la calidad percibida, se relaciona con la respuesta subjetiva del consumidor hacia las características o atributos de una marca o producto. El consumidor percibe el producto como un conjunto de atributos que pueden ser empleados como indicadores para inferir su calidad (Keller, 1993). Una elevada calidad percibida tiene lugar cuando los consumidores reconocen la diferenciación y superioridad de una marca en relación a otras marcas competidoras. Esta calidad percibida influirá en sus decisiones de compra y en su elección de marca, escogiendo aquellas en las que se percibe una mayor calidad. De acuerdo con Zeithaml (1988), la calidad percibida es el resultado global de diferentes estímulos que el consumidor puede emplear para evaluar la calidad de una marca, entre los cuales se incluyen los atributos extrínsecos del producto, como la marca, los ingredientes o el país de origen.

En cuanto a las asociaciones de marca -o imagen de marca-, se pueden definir como aquel conjunto de asociaciones que están unidas a la marca en la mente del consumidor (Aaker, 1991, 1996). Siguiendo a Yoo et al., (2000), las asociaciones de marca consisten en aquel conjunto de imágenes, ideas, hechos o cualquier elemento que haya formado una relación sólida con el conocimiento de la marca. Mediante las asociaciones de marca, las compañías pueden diferenciar y posicionar sus productos en el mercado, creando actitudes y sentimientos positivos hacia una marca (Aaker, 1991; Dean, 2004), y esto puede traducirse en una mayor predisposición a adquirir el producto (Yoo et al., 2000; Chen, 2001).

Por último, la variable lealtad de marca refleja en grado de satisfacción del consumidor con la marca y puede generar compromiso y fidelidad hacia una marca concreta (Aaker, 1991). Numerosos estudios han comprobado que la lealtad del consumidor hacia una marca es uno de los principales factores que influye de manera positiva y directa en el valor de marca (Yoo et al., 2000; Atilgan et al., 2005). Esto se debe a que los consumidores leales a una marca, muestran respuestas más favorables y positivas hacia la marca, que aquellos 
no leales (Grover y Srinivasan, 1992). Por lo tanto, nuestro estudio comprende un modelo de formación del valor de marca que incorpora las cuatro dimensiones propuestas por Aaker (1991), como son la notoriedad de marca, las asociaciones de marca, la lealtad de marca y la calidad percibida.

Sin embargo, nuestro estudio pretende analizar también las consecuencias que el valor de marca tiene en el comportamiento del consumidor, para lo cual hemos incluido el análisis de otras dos dimensiones del valor de marca, como son la disposición del consumidor a pagar un precio primado por el producto o la marca (Netemeyer et al., 2004) y la intención de compra del consumidor (Burke y Schoeffler, 1980; Wilson, 1981). La literatura previa señala que el valor de marca influye en la disposición de los consumidores a pagar un precio primado (Arvidsson, 2006). Numerosos autores han demostrado que los consumidores están dispuestos a pagar un precio mayor por las marcas que poseen asociaciones positivas y favorables, o bien por aquellas marcas que ofrecen una mayor calidad (Erdem et al., 2002; Netemeyer et al., 2004). De la misma manera, el valor de marca se ha considerado como una condición para la elección o preferencia de una marca, que en consecuencia, afecta a la intención de compra. Varios estudios señalan la relación positiva entre las dimensiones del valor de marca, la preferencia de marca y la intención de compra (Cobb-Walgren et al., 1995; Myers, 2003).

Por último, debemos hacer referencia al concepto de país de origen del producto, dado que una de las marcas analizadas es mexicana. El concepto de país de origen se refiere a las opiniones que tienen los consumidores acerca de las características de los productos y servicios producidos en diferentes países (Bilkey, 1993). Un gran número de investigadores han analizado la importancia de la imagen país de origen en el comportamiento del consumidor, destacando su influencia en la valoración general del producto, como señal de otras características intrínsecas del producto que no pueden evaluarse directamente (Bilkey y Nes, 1982; Han y Terpstra, 1988; Yoo, 1992). Rao y Monroe (1989) señalan que en aquellos productos que son poco familiares para el consumidor, suponen un menor empleo de las variables intrínsecas para su valoración. Por 
tanto, para productos y marcas importadas -y por tanto, menos familiares-, los consumidores confiarán en la marca y el país de origen. Más concretamente, en relación al sector de la comida y bebida, los consumidores emplean la información acerca del país de origen, como un indicador de la calidad de los productos importados (Verbeke y Ward, 2006; Dekhili y d'Hauteville, 2009).

\section{OBJETIVO}

Nuestro estudio persigue dos objetivos. En primer lugar, analizar de manera descriptiva el perfil del consumidor de cerveza, en cuanto a su frecuencia y lugar de consumo y los atributos que valora en el producto. En segundo lugar, nuestro trabajo pretende analizar el valor de marca en el sector de la cerveza, y más concretamente si existen diferencias significativas entre el valor de marca que posee una marca de cerveza nacional y otra marca de cerveza importada. Para la segunda parte del trabajo, seleccionamos dos marcas presentes en el mercado, de entre las que fueron escogidas por los consumidores como aquellas con mayor frecuencia de consumo. Seleccionamos una marca nacional -Mahou- y otra marca importada de México-Coronita-, con el objetivo de estudiar si los consumidores españoles perciben diferencias en cuanto al valor de marca de ambas, e incluso si estarían dispuestos a pagar un precio primado por ellas, así como analizar cuál es su intención de compra para ambas marcas. Por lo tanto, nuestro objetivo es analizar las posibles diferencias en el valor de marca y explicar cuales son las diferencias existentes, en el caso de resultar significativas.

\section{METODOLOGIA}

\subsection{LA SELECCIÓN DE MARCAS}

Este trabajo se ha basado en los datos obtenidos a través de la realización de una encuesta entre consumidores residentes en España. Los datos fueron recogidos durante el mes de marzo de 2012, mediante un cuestionario que se les hizo llegar mediante correo electrónico. En este primer cuestionario 
exploratorio, se incluyeron algunas preguntas para conocer los hábitos de consumo de cerveza y las preferencias de los consumidores, como son la frecuencia de consumo, el lugar de consumo, los atributos o variables que más se valoran en el producto, así como el tipo de cerveza que se consume. Por último se incluyeron algunas variables socio demográficas como el sexo y la edad. Se obtuvieron un total de 281 cuestionarios válidos, que se utilizaron para la primera parte del trabajo, esto es, para el análisis descriptivo. En este primer cuestionario, también se pretendía conocer cuáles son las marcas que los españoles consumen con mayor frecuencia, de entre una serie de marcas sugeridas, todas ellas presentes en el mercado español, para seleccionar las marcas objetivo de la segunda parte de nuestro trabajo. El resultado obtenido fue que las marcas con mayor frecuencia de consumo son Mahou -marca española- y Estrella Galicia -marca española con una gran implantación en algunas regiones españolas-, así como tres marcas importadas en el mercado español, Heineken, Coronita y Calsberg. Finalmente, como marca nacional se seleccionó la marca Mahou, por ser propiedad de la mayor empresa cervecera española, y porque junto con Estrella Galicia, Cruzcampo y San Miguel tiene la mayor frecuencia de consumo. En cuanto a la marca importada, se seleccionó la marca Coronita. El motivo de esta elección es que como marcas importadas con mayor consumo se citaron Heineken, Carlsberg y la propia Coronita, y con nuestra elección de marca buscábamos un nombre de marca castellano, es decir, una marca de cerveza que no tuviera un nombre de marca extranjero. Para la segunda parte del estudio, se obtuvieron un total de 100 cuestionarios válidos para cada una de las dos marcas analizadas.

\subsection{LA SELECCION DE VARIABLES}

Para la selección de las variables, se han empleado ítems propuestos en la bibliografía más relevante sobre el tema. En primer lugar, para medir la notoriedad de marca se emplearon 5 ítems propuestos por Yoo et al. (2000) y Netemeyer et al. (2004), los cuales se refieren al conocimiento global que el consumidor tiene de la marca, a su capacidad para identificarla y reconocerla 
frente a otras marcas de la competencia, así como la familiaridad del consumidor con la marca. En segundo lugar, la calidad percibida se midió empleando cuatro ítems, empleados por Pappu et al. (2005, 2006), los cuales analizan la calidad percibida de manera general, dejando a un lado los atributos específicos de la categoría de producto. En relación a la lealtad de marca, se empleó la escala utilizada por Yoo et al. (2000), la cual valora si un consumidor se considera leal a una marca y si la marca por la que se le pregunta sería su primera opción de compra. En relación a las asociaciones de marca, se consideraron tres tipos de asociaciones (Aaker, 1996), como son el valor percibido, la personalidad de la marca y las asociaciones de carácter organizacional. Estos ítems se analizaron a través de las escalas propuestas por varios autores (Lassar et al., 1995; Aaker, 1996; Netemeyer et al., 2004, Pappu et al., 2005, 2006). Para medir la intención a pagar un precio primado por la marca, se empleó la propuesta por Netemeyer et al. (2004) y para analizar la intención de compra del consumidor, se han utilizado los ítems propuestos por Wilson (1981). Finalmente, siguiendo la propuesta realizada por Yoo et al. (2000) se ha incluido en nuestro trabajo una variable que recoge el valor de marca global, que se puede definir como la superioridad de una marca frente a otras marcas y alternativas similares. Para el desarrollo de las escalas de medida hemos utilizado las escalas clásicas de tipo Likert de cinco puntos, siendo 1 totalmente en desacuerdo y 5 totalmente de acuerdo.

\subsection{TRABAJO DE CAMPO Y ANALISIS DE DATOS}

El universo de nuestro estudio son los consumidores residentes en España. El tamaño de la muestra ha sido de 281 cuestionarios válidos y el tipo de encuesta ha sido electrónica. El trabajo de campo se realizó durante el mes de marzo de 2012, mediante un muestreo aleatorio, no estratificado. Esta muestra fue empleada para la primera parte del trabajo, esto es, el análisis descriptivo del consumidor. También se empleó esta información para analizar cuáles eran las marcas de cerveza más consumidas, seleccionando de esta manera dos marcas para la segunda parte del estudio, el análisis comparativo de su valor 
de marca. Para esta segunda parte del trabajo se obtuvieron 100 cuestionarios válidos de cada una de las marcas analizadas- Mahou y Coronita-. De nuevo, el tipo de encuesta empleada fue electrónica.

En nuestro estudio se han empleado dos tipos de técnicas y de análisis de datos claramente diferenciados. Para la primera parte del trabajo, y con la intención de obtener un análisis descriptivo de los hábitos y preferencias de los consumidores de cerveza se ha llevado a cabo una distribución de frecuencias relativa. Por otro lado, para la segunda parte de nuestro estudio, esto es, para el análisis comparativo de las dos marcas de cerveza seleccionadas se realizarán dos análisis diferentes. En primer lugar, se llevará a cabo un análisis de los valores medios y desviaciones típicas. En segundo lugar, y dado que nuestro objetivo es también estudiar las posibles diferencias de valor de marca por parte de los consumidores de cerveza españoles, en el caso de que las hubiese. Por ese motivo, las técnicas estadísticas utilizadas en esta segunda parte de la investigación han consistido en el análisis de tablas de contingencia, aplicando el análisis de comparación de medias para dos muestras independientes - realizado previamente el test de Levene-, de manera que se pueden comparar las diferencias para cada uno de los ítems estudiados y analizar aquellas diferencias que son estadísticamente significativas.

\section{RESULTADOS}

\subsection{ANALISIS DESCRIPTIVO}

En primer lugar, observamos que la variable notoriedad de marca, alcanza valores más altos para todos los ítems analizados para la marca nacional Mahou-, lo cual parece lógico al tratarse de una marca con gran implantación en España y por tratarse del primer grupo productor de cerveza nacional. Sin embargo, encontramos que para el ítem NOT5 Puedo reconocer la marca Coronita frente a otras marcas de cerveza competidoras, la marca Coronita alcanza un valor mayor (una media de 3.88), lo cual indica que se trata de una 
marca que los consumidores españoles identifican y reconocen con facilidad. En relación a la variable calidad percibida de la marca, se han obtenido valores superiores para la marca Coronita para todos los ítems analizados. Se debe destacar, en este punto, que para el ítem CALPER2 Los productos de la marca tienen una calidad consistente, se alcanza un valor medio de 3.48 para la marca Coronita, lo cual muestra que los consumidores perciben que dicha marca de importación ofrece un producto con una calidad sin cambios ni modificaciones a lo largo del tiempo.

Al analizar la variable asociaciones de marca -o imagen de marca-, merecen destacarse los siguientes resultados. En primer lugar, en cuanto al valor percibido por el consumidor, los resultados muestran que la marca Mahou posee para la mayoría de los ítems estudiados, una mejor valoración que la marca Coronita. Sin embargo, los valores medios obtenidos por ambas marcas son muy similares. Únicamente para el ítem ASO2 Dentro del mercado de cerveza, considero que la marca $X$ es una buena compra, Coronita obtiene una mejor valoración. En segundo lugar, merece ser destacado que para la variable personalidad de marca, la marca importada Coronita obtiene una valoración superior en comparación a la marca nacional Mahou. En este punto, se debe destacar la valoración media de 3.56 para el ítem ASO4 La marca tiene personalidad, lo que indica que los consumidores españoles perciben la marca Coronita como una marca que les resulta interesante y con gran personalidad. En último lugar, en cuanto a la variable asociaciones organizacionales, esto es, la imagen que el consumidor tiene de la empresa fabricante del producto, no existen grandes diferencias entre ambas marcas.

En relación a la lealtad de marca, también se obtienen mejores valoraciones en todos los ítems analizados para la marca importada Coronita, resultando interesante destacar que para el ítem LEA3 No compraría otras marcas de cerveza si la marca Coronita está disponible en el punto de venta la marca Coronita obtiene un valor medio de 2.04, frente al valor medio de 1.54 que obtiene la marca Mahou. $Y$ en cuanto al valor de marca general o total que la enseña comercial tiene para la categoría de producto, se debe señalar que de 
nuevo la marca Coronita obtiene las mejores valoraciones por parte de los consumidores españoles, y para todos los ítems analizados. Por último, para la disposición a pagar un precio primado, encontramos que en general las valoraciones medias son muy bajas, lo que refleja que el consumidor español tiene una baja disposición a pagar un precio primado para cualquiera de las dos marcas de cerveza analizadas, siendo más baja para la marca nacional Mahou. Aquí, debemos destacar que la marca nacional obtiene una valoración muy baja, de 1.31 para el ítem PRE2 Estoy dispuesto a pagar un precio mayor por la marca Mahou que por otras marcas de cerveza. Por último, se ha analizado la variable intención de compra, y en este caso, los resultados señalan también una mejor valoración para la marca importada Coronita, en todos los ítems analizados. En este punto, se debe mencionar que para el ítem INT1 Compraría cerveza de la marca, Coronita obtiene un valor medio de 2.92 frente al valor medio de 2.18 de la marca Mahou; e incluso para el ítem INT2 Definitivamente, consideraría comprar la marca, también la marca Coronita alcanza una mejor valoración (2.87) frente a la marca nacional (2.12), lo cual parece apuntar a una mayor disposición de los consumidores españoles a adquirir la marca Coronita en el mercado, frente a la marca nacional.

Tabla 1. Indicadores, valores medios y desviación típica de las dimensiones de Valor de marca.

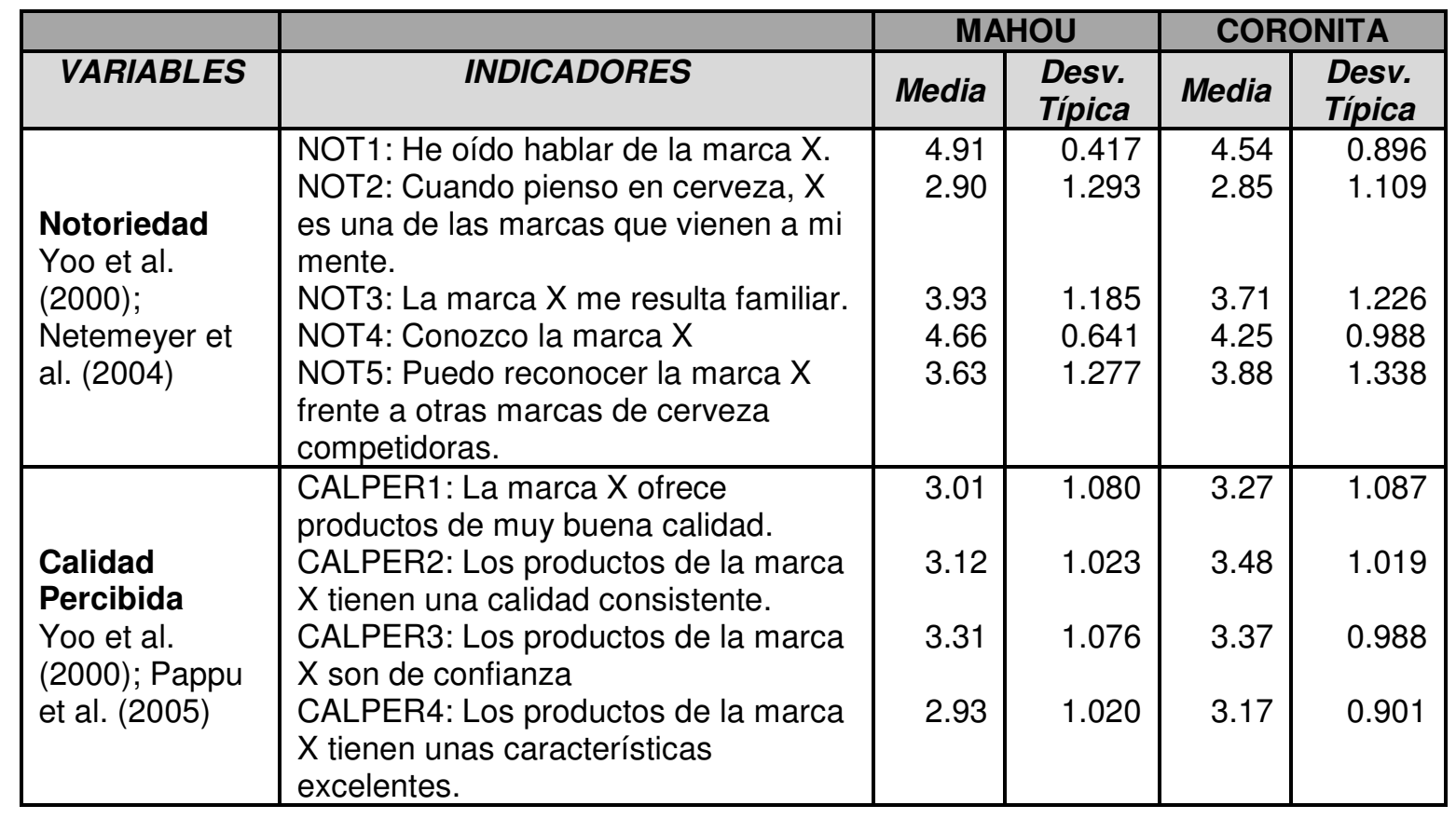




\begin{tabular}{|c|c|c|c|c|c|}
\hline $\begin{array}{l}\text { Valor } \\
\text { Percibido } \\
\text { Lassar et al. } \\
\text { (1995), Aaker } \\
\text { (1996), } \\
\text { Netemeyer et } \\
\text { al. (2004) }\end{array}$ & $\begin{array}{l}\text { ASO1: La marca X tiene una buena } \\
\text { relación calidad-precio. } \\
\text { ASO2: Dentro del Mercado de } \\
\text { cerveza, considero que la marca X es } \\
\text { una buena compra. } \\
\text { ASO3: La marca X aporta un elevado } \\
\text { valor en relación al precio que hay que } \\
\text { pagar por ella. }\end{array}$ & $\begin{array}{l}2.84 \\
2.82 \\
2.73\end{array}$ & $\begin{array}{l}0.898 \\
1.058\end{array}$ & $\begin{array}{l}2.75 \\
3.02\end{array}$ & $\begin{array}{l}1.082 \\
1.093\end{array}$ \\
\hline $\begin{array}{l}\text { Personalidad } \\
\text { de marca } \\
\text { Aaker (1996) }\end{array}$ & $\begin{array}{l}\text { ASO4: La marca X tiene personalidad. } \\
\text { ASO5: La marca X es interesante. } \\
\text { ASO6: Tengo una imagen clara del } \\
\text { tipo de personas que consumen la } \\
\text { marca X. }\end{array}$ & $\begin{array}{l}2.88 \\
2.58 \\
2.12\end{array}$ & $\begin{array}{l}1.148 \\
1.061 \\
1.108\end{array}$ & $\begin{array}{l}3.56 \\
3.42 \\
2.63\end{array}$ & $\begin{array}{l}1.110 \\
0.957 \\
1.237\end{array}$ \\
\hline $\begin{array}{l}\text { Asociaciones } \\
\text { Organizaciona } \\
\text { les } \\
\text { Aaker (1996), } \\
\text { Pappu et al. } \\
(2005,2006)\end{array}$ & $\begin{array}{l}\text { ASO7: Confío en la empresa que } \\
\text { fabrica la marca X. } \\
\text { ASO8: Me gusta la empresa que } \\
\text { fabrica la marca X. } \\
\text { ASO9: La empresa que fabrica la } \\
\text { marca X tiene credibilidad. }\end{array}$ & $\begin{array}{l}2.88 \\
2.70 \\
2.12\end{array}$ & $\begin{array}{l}1.148 \\
1.059 \\
1.052\end{array}$ & $\begin{array}{l}2.85 \\
2.69 \\
2.98\end{array}$ & $\begin{array}{l}1.017 \\
0.961 \\
0.960\end{array}$ \\
\hline $\begin{array}{l}\text { Lealtad } \\
\text { Yoo et al. } \\
\text { (2000) }\end{array}$ & $\begin{array}{l}\text { LEA1: Me considero un consumidor } \\
\text { leal a la marca X. } \\
\text { LEA2: Si comprara cerveza, X sería mi } \\
\text { primera opción de compra. } \\
\text { LEA3: No compraría otras marcas de } \\
\text { cerveza si la marca X estuviera } \\
\text { disponible en el punto de venta. }\end{array}$ & $\begin{array}{l}1.55 \\
1.70 \\
1.54\end{array}$ & $\begin{array}{l}0.858 \\
1.030 \\
0.910\end{array}$ & $\begin{array}{l}1.88 \\
1.90 \\
2.04\end{array}$ & $\begin{array}{l}1.078 \\
1.015 \\
1.137\end{array}$ \\
\hline $\begin{array}{l}\text { Valor de } \\
\text { Marca } \\
\text { Yoo et al. } \\
(2000)\end{array}$ & $\begin{array}{l}\text { VM1: Tiene sentido comprar la marca } \\
\text { X en lugar de otras marcas de cerveza } \\
\text { aunque sean iguales. } \\
\text { VM2: Aun habiendo otras marcas de } \\
\text { cerveza con características similares a } \\
\text { la marca X, yo prefiero comprar la } \\
\text { marca X. } \\
\text { VM3: Aunque hubiera otras marcas de } \\
\text { cerveza tan buenas como X, yo } \\
\text { prefería comprar la marca X. } \\
\text { VM4: Aunque la marca X no fuera } \\
\text { diferente a otras marcas de cerveza, } \\
\text { es más inteligente comprar la marca } \\
\text { X. }\end{array}$ & $\begin{array}{l}1.63 \\
1.88\end{array}$ & $\begin{array}{l}0.850 \\
0.993\end{array}$ & 2.62 & $\begin{array}{l}1.103 \\
1.060\end{array}$ \\
\hline $\begin{array}{l}\text { Disposición a } \\
\text { pagar un } \\
\text { precio } \\
\text { primado } \\
\text { Netemeyer et } \\
\text { al. (2004) }\end{array}$ & $\begin{array}{l}\text { PRE1: El precio de la marca X tendría } \\
\text { que subir bastante para que } \\
\text { considerara no comprarla. } \\
\text { PRE2: Estoy dispuesto a pagar un } \\
\text { precio mayor por la marca X que por } \\
\text { otras marcas de cerveza. } \\
\text { PRE3: Estoy dispuesto a pagar mucho } \\
\text { más por la marca X que por otras } \\
\text { marcas de cerveza. }\end{array}$ & $\begin{array}{l}1.34 \\
1.31\end{array}$ & $\begin{array}{l}0.617 \\
0.583\end{array}$ & $\begin{array}{l}2.25 \\
1.87\end{array}$ & $\begin{array}{l}1.046 \\
0.991\end{array}$ \\
\hline $\begin{array}{l}\text { Intención de } \\
\text { compra } \\
\text { Wilson (1981) }\end{array}$ & $\begin{array}{l}\text { INT1: Compraría cerveza de la marca } \\
\text { X. } \\
\text { INT2: Definitivamente, consideraría } \\
\text { comprar la marca X. } \\
\text { INT3: Es muy probable que compre la } \\
\text { marca X. }\end{array}$ & $\begin{array}{l}2.18 \\
2.12 \\
2.34\end{array}$ & $\begin{array}{l}1.141 \\
1.080 \\
1.746\end{array}$ & 2.92 & $\begin{array}{l}1.266 \\
1.253\end{array}$ \\
\hline
\end{tabular}

Fuente: Elaboración propia. 


\subsection{ANALISIS DE COMPARACION DE MEDIAS}

Dado que el objetivo de nuestro trabajo es analizar las posibles diferencias de percepción del valor de marca por parte de los consumidores de cerveza españoles, hemos procedido a testar las hipótesis de diferencias de medias para dos muestras independientes, mediante el estadístico $t$ de student y el correspondiente test de Levene. De esta manera hemos podido comparar las diferencias existentes, para cada uno de los ítems estudiados y se han podido analizar aquellas diferencias que son estadísticamente significativas. Para cada una de las variables analizadas, consideramos como hipótesis nula la siguiente:

$H_{0}$ : No existen diferencias significativas entre la marcas de cerveza Mahou y Coronita

\subsubsection{Notoriedad de marca.}

Para analizar cómo los consumidores españoles perciben la notoriedad de marca para las dos marcas de cerveza analizadas, partimos de la siguiente hipótesis nula:

$\mathrm{H}_{01}$ : No existen diferencias significativas en Notoriedad de marca para las marcas analizadas, es decir, los consumidores tienen la misma capacidad de identificar y reconocer las dos marcas.

Tabla 2: Comparación de medias para la dimensión Notoriedad.

\begin{tabular}{lrrcrc}
\multicolumn{1}{c}{$\begin{array}{c}\text { t medias muestras } \\
\text { independientes }\end{array}$} & $\mathbf{F}$ & Sig. & t & gl & $\begin{array}{c}\text { Sig. } \\
\text { (bilateral) }\end{array}$ \\
\hline NOT1 $\left({ }^{*}\right)$ & 32.71 & 0.00 & -2.771 & 68.809 & 0.007 \\
NOT2 $\left({ }^{*}\right)$ & 5 & 1 & & & \\
& 1.172 & 0.28 & -0.220 & 117 & 0.827 \\
NOT3 $\left({ }^{*}\right)$ & 1.281 & 0.26 & & & \\
& 16.962 & 0.00 & & & \\
NOT4 $\left({ }^{*}\right)$ & 1 & 1 & -2.578 & 82.931 & 0.338 \\
NOT5 $\left({ }^{*}\right)$ & 0.046 & 0.83 & 1.069 & 117 & 0.012 \\
& 1 & 1 & & &
\end{tabular}


$\left({ }^{*}\right)$ Se han asumido varianzas iguales

$\left({ }^{* *}\right)$ Se han asumido varianzas diferentes

Se rechaza la hipótesis nula de igualdad de medias para los ítems NOT1 He oído hablar de la marca y NOT4 Conozco la marca, de manera que podemos afirmar que existen diferencias significativas entre las dos marcas analizadas, Mahou y Coronita con un nivel de significación del $0.05 \%$. En este caso, la marca nacional Mahou obtiene una mejor valoración para ambos ítems, tal y como refleja la tabla 2. Por tanto, podemos se puede rechazar que la notoriedad de marca sea igual para ambas marcas de cerveza, afirmando que la notoriedad es superior para la marca Mahou que para la marca Coronita.

\subsubsection{Calidad Percibida.}

$\mathrm{H}_{02:}$ No existen diferencias significativas en calidad percibida para las marcas analizadas, esto es, los consumidores perciben que tienen una calidaa similar.

Tabla 3: Comparación de medias para la dimensión Calidad Percibida

\begin{tabular}{lcrccc}
\multicolumn{1}{c}{$\begin{array}{c}\text { t medias muestras } \\
\text { independientes }\end{array}$} & F & Sig. & t & gl & $\begin{array}{c}\text { Sig. } \\
\text { (bilateral) }\end{array}$ \\
\hline CALPER1 $\left(^{*}\right)$ & 0.899 & 0.34 & 1.271 & 117 & 0.206 \\
CALPER2 $\left(^{*}\right)$ & 0.126 & 0.72 & 1.915 & 117 & 0.058 \\
& 3 & & & \\
CALPER3 $\left(^{*}\right)$ & 1.042 & 0.31 & 0.279 & 117 & 0.780 \\
& 1.843 & 0.17 & 1.382 & 117 & 0.170 \\
CALPER4 $\left(^{*}\right)$ & 7 & & & \\
$\begin{array}{l}\left(^{*}\right) \text { Se han asumido varianzas iguales } \\
\left({ }^{*}\right) \text { Se han asumido varianzas diferentes }\end{array}$ & & & &
\end{tabular}

Se acepta la hipótesis nula de igualdad de medias para todos los ítems de calidad percibida, de manera que podemos afirmar que no existen diferencias significativas entre las dos marcas analizadas, Mahou y Coronita con un nivel de significación del $0.05 \%$. Es decir, los 
consumidores españoles perciben que no hay diferencias en cuanto a la calidad del producto para las dos marcas analizadas. Sin embargo, la calidad percibida es superior para la marca Coronita si atendemos a los valores medios obtenidos para los ítems analizados.

\subsubsection{Asociaciones de marca.}

$\mathrm{H}_{03}$ : No existen diferencias significativas en Imagen de marca para las marcas analizadas, esto es, los consumidores perciben que tienen una imagen de marca similar.

Tabla 4: Comparación de medias para la dimensión asociaciones de marca

\begin{tabular}{lrrrrr}
$\begin{array}{c}\text { t medias muestras } \\
\text { independientes }\end{array}$ & F & Sig. & t & gl & $\begin{array}{c}\text { Sig. } \\
\text { (bilateral) }\end{array}$ \\
\hline ASO1 $\left(^{* *}\right)$ & 4.544 & 0.03 & -0.462 & 98.337 & 0.645 \\
ASO2 $\left(^{*}\right)$ & 0.007 & 0.93 & 1.000 & 117 & 0.320 \\
& 2 & & & \\
ASO3 $\left(^{*}\right)$ & 0.042 & 0.83 & -0.220 & 117 & 0.827 \\
& 0.046 & 0.83 & 3.237 & 117 & 0.002 \\
& 0.671 & 1 & 4.475 & 117 & 0.001 \\
ASO4 $\left(^{*}\right)$ & 0.857 & 0.41 & 2.391 & 117 & 0.018 \\
ASO5 $\left(^{*}\right)$ & 1.335 & 4 & -0.170 & 117 & 0.865 \\
ASO6 $\left(^{*}\right)$ & 0.081 & 0.35 & -0.049 & 117 & 0.961 \\
ASO7 $\left(^{*}\right)$ & 1.833 & 6 & 4.602 & 117 & 0.001 \\
ASO8 $\left(^{*}\right)$ & & 0.25 & & & \\
ASO9 $\left(^{*}\right)$ & 0.75 & & & \\
& & 0.77 & & & \\
& & 6 & & &
\end{tabular}

$\left({ }^{*}\right)$ Se han asumido varianzas iguales

$\left({ }^{* *}\right)$ Se han asumido varianzas diferentes.

Se rechaza la hipótesis nula de igualdad de asociaciones de marca para los ítems ASO4 La marca tiene personalidad, ASO5 La marca es interesante, ASO6 Tengo una imagen clara del tipo de personas que consumen la marca y para ASO9 La empresa que fabrica la marca tiene credibilidad, para los cuales existen diferencias significativas entre las dos marcas con un nivel de significación del $0.05 \%$. Se puede afirmar que la marca Coronita 
obtiene una mejor valoración y por tanto, podemos rechazar que las asociaciones de marca o imagen de marca sea similar para las dos marcas de cerveza analizadas.

\subsubsection{Lealtad de marca}

$\mathrm{H}_{04}$ : No existen diferencias significativas en Lealtad de marca para las marcas analizadas, esto es, los consumidores tienen una fidelidad hacia la marca similar para ambas.

Tabla 5: Comparación de medias para la dimensión Lealtad t medias muestras independientes $\quad F \quad$ Sig. $\quad$ t $\quad$ gl $\begin{gathered}\text { Sig. } \\ \text { (bilateral) }\end{gathered}$

\begin{tabular}{llllll}
\hline LEA1 $\left(^{*}\right)$ & 3.157 & 0.078 & 1.873 & 117 & 0.064 \\
LEA2 $\left(^{*}\right)$ & 0.094 & 0.760 & 1.070 & 117 & 0.287 \\
LEA3 $\left(^{*}\right)$ & 3.869 & 0.052 & 2.671 & 117 & 0.009
\end{tabular}

$\left.{ }^{*}\right)$ Se han asumido varianzas iguales

$\left.{ }^{* *}\right)$ Se han asumido varianzas diferentes

Se rechaza la hipótesis nula de igualdad de Lealtad de marca para el ítem LEA3 No compraría otras marcas de cerveza si la marca estuviera disponible en el punto de venta, para el cual existen diferencias significativas entre las dos marcas con un nivel de significación del $0.05 \%$. En este caso, podemos afirmar que la marca importada Coronita obtiene una mejor valoración, de manera que los consumidores españoles no comprarían otra marca de cerveza si en el punto de venta estuviera disponible Coronita.

\subsubsection{Valor de marca.}

$\mathrm{H}_{05}$ : No existen diferencias significativas en Valor de marca para las marcas analizadas, esto es, los consumidores perciben que ambas marcas poseen un valor similar.

Tabla 6: Comparación de medias para la dimensión Valor de marca

\begin{tabular}{lcccccc}
$\begin{array}{c}\text { t medias muestras } \\
\text { independientes }\end{array}$ & F & Sig. & t & gl & $\begin{array}{c}\text { Sig. } \\
\text { (bilateral } \\
\text { ) }\end{array}$ \\
\hline $\mathrm{VM} 1\left(^{*}\right)$ & 1.137 & 0.28 & 3.83 & 117 & 0.001
\end{tabular}




\begin{tabular}{lrrrrr}
\hline & & 9 & 5 & & \\
VM2 $\left(^{* *}\right)$ & 13.58 & 0.00 & 4.42 & 91.42 & 0.001 \\
VM3 $\left(^{* *}\right)$ & 1 & 1 & 3 & 6 & \\
& 5.821 & 0.01 & 3.99 & 93.48 & 0.001 \\
VM4 $\left(^{*}\right)$ & & 5 & 4 & \\
$\left(^{*}\right)$ Se han asumido varianzas iguales & 0.046 & 0.83 & 1.24 & 117 & 0.217 \\
$\left(^{* *}\right.$ Se han asumido varianzas diferentes & & & 2 & & \\
\end{tabular}

Se rechaza la hipótesis nula de igualdad de Valor de marca para los ítems VM1 Tiene sentido comprar la marca en lugar de otras marcas de cerveza aunque sean iguales, VM2 Aun habiendo otras marcas de cerveza con características similares a la marca, yo prefiero comprarla, y para VM3 Aunque hubiera otras marcas de cerveza tan buenas como ésta, yo preferiría comprar la marca, para los cuales existen diferencias significativas entre las dos marcas analizadas con un nivel de significación del $\mathbf{0 . 0 5 \%}$. Por lo tanto, podemos afirmar que la marca importada Coronita obtiene un mayor valor de marca para los consumidores de cerveza españoles que la marca nacional Mahou, entendido como valor de marca general o total de la marca o enseña comercial.

\subsubsection{Disposición a pagar un precio primado.}

$\mathrm{H}_{06}$ : No existen diferencias significativas en Disposición a pagar un precio primado para las marcas analizadas.

Tabla 7: Comparación de medias para la dimensión Disposición a pagar un precio primado

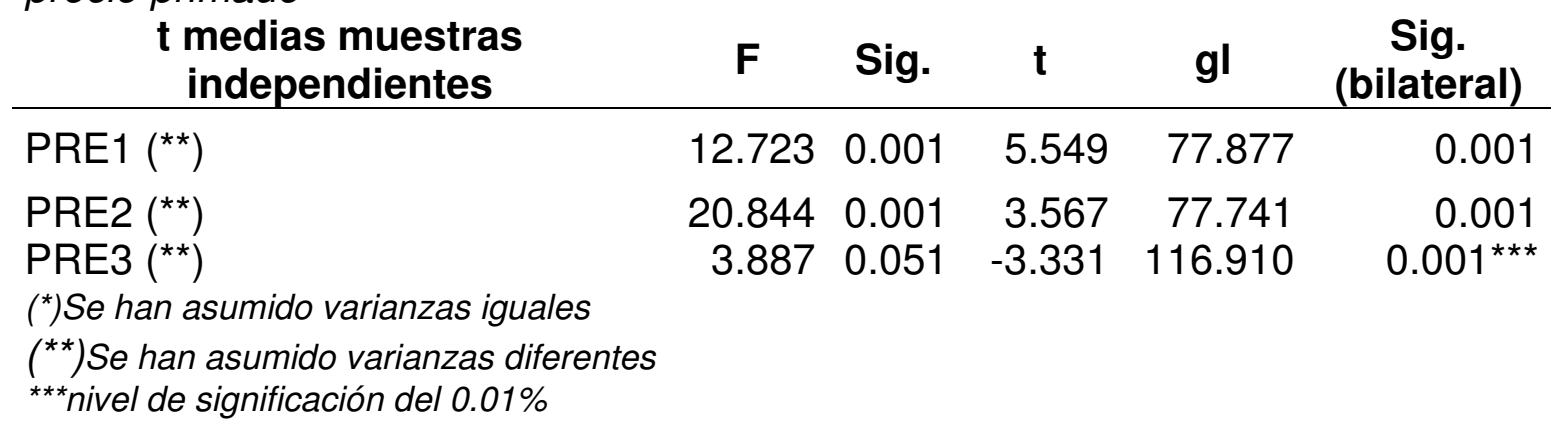

Se rechaza la hipótesis nula para todos los ítems analizados. De esta manera, podemos afirmar que existen diferencias significativas, con un nivel de 
significación del $0.05 \%$, para los ítems PRE1 El precio de la marca tendría que subir bastante para que considerara no comprarla y para PRE2 Estoy dispuesto a pagar un precio mayor por la marca, que por otras marcas de cerveza; mientras que el nivel de significación es del $0.10 \%$ para el ítem PRE3 Estoy dispuesto a pagar mucho más por la marca, que por otras marcas de cerveza. Se puede concluir que los consumidores españoles no tienen una disposición a pagar un precio primado, similar para ambas marcas, dado que tal y como se señala en la tabla 2, esta disposición es mayor para la marca Coronita.

\subsubsection{Intención de compra.}

$\mathrm{H}_{07}$ : No existen diferencias significativas en Intención de compra para las marcas analizadas, esto es, los consumidores poseen una intención de compra similar.

Tabla 8: Comparación de medias para la dimensión Intención de compra

\begin{tabular}{lrrrrr}
\multicolumn{1}{c}{$\begin{array}{c}\text { t medias muestras } \\
\text { independientes }\end{array}$} & F & Sig. & t & gl & $\begin{array}{c}\text { Sig. } \\
\text { (bilateral }\end{array}$ \\
\hline INT1 $\left(^{*}\right)$ & 0.219 & 0.64 & 3.36 & 117 & 0.001 \\
& & 1 & 4 & & \\
INT2 $\left(^{*}\right)$ & 0.963 & 0.32 & 3.48 & 117 & 0.001 \\
& 18.10 & 0.00 & 1.39 & 116.71 & 0.166 \\
INT3 $\left(^{* *}\right)$ & 2 & 1 & 4 & 1 & \\
$\left(^{*}\right)$ Se han asumido varianzas iguales & & & & & \\
$\left(^{*}\right)$ Se han asumido varianzas diferentes & & & & &
\end{tabular}

Se rechaza la hipótesis nula de igualdad de Intención de compra para los ítems INT1 Compraría la marca de cerveza e INT2 Definitivamente, consideraría comprar la marca, con un nivel de significación del $0.05 \%$. Si se analizan los valores medios obtenidos para cada una de las marcas, podemos afirmar que los consumidores tienen una mayor Intención de compra hacia la marca de cerveza importada Coronita, y que esta intención de compra es menor para la marca nacional Mahou, lo cual estaría relacionado con el distinto valor de marca que ambas poseen para los consumidores. 
Los resultados obtenidos, ponen de manifiesto que la marca española Mahou, únicamente obtiene una mejor valoración de la notoriedad de marca, lo cual tiene cierta lógica, dado que se trata de una marca nacional y con una implantación mayor en el mercado español. Por otro lado, resulta destacable que la marca importada Coronita, obtiene una valoración superior para el resto de las variables de valor de marca, esto es, para la calidad percibida, las asociaciones de marca y la lealtad de marca. Asimismo, obtiene una mejor valoración del valor de marca entendido de manera general y para las consecuencias del valor de marca analizadas, es decir, la disposición a pagar un precio primado y la intención de compra del producto.

\section{CONCLUSIONES}

Si bien el valor de marca ha recibido una atención continuada por parte de los investigadores y los gestores de marketing, y existe una gran abundancia de modelos y conceptos relacionados con el valor de marca, existen pocos estudios comparativos basados en datos empíricos. En esta situación, el presente trabajo supone un análisis del valor de la marca en un mercado y para un producto concreto, la cerveza. En relación a la primera parte del trabajo, podemos concluir, que la mayoría de los consumidores españoles beben cerveza con bastante frecuencia y regularidad, y que respecto al lugar de consumo - a pesar de que los últimos datos ponen de relieve un repunte del consumo en el hogar debido a la reciente crisis económica- sigue siendo mayoritario el consumo en el canal Horeca, es decir, bares, cafeterías y terrazas. Debe subrayarse que en cuanto a los atributos que más valoran los consumidores españoles de este producto, destaca el sabor, por encima de otras cualidades como el aroma o la textura. Este resultado, debería ser tenido en consideración por parte de las compañías y los gestores de marketing de las empresas cerveceras a la hora de desarrollar sus campañas de comunicación y publicidad, reforzando y comunicando dicha característica de su producto; o bien empleando el sabor como una variable para el posicionamiento de la marca en el mercado. 
En segundo lugar, y en relación a la percepción del valor de marca por parte de los consumidores, para las dos marcas de cerveza comparadas, podemos concluir que la marca de importación -Coronita- tiene un mayor valor de marca que la cerveza nacional. Se debe destacar que la marca importada Coronita, obtiene una valoración superior para todos los componentes o fuentes del valor de marca, excepto para la notoriedad. En este punto, la marca nacional Mahou - muestra una mejor valoración de la notoriedad de marca, lo cual parece lógico al tratarse de una marca de la mayor empresa cervecera española y con una gran implantación en el mercado nacional. Sin embargo, los resultados obtenidos ponen de manifiesto que la marca de cerveza Coronita obtiene una mejor valoración para las asociaciones de marca, la calidad percibida y la lealtad de marca. Por último, la marca Coronita, obtiene una mejor valoración de marca general, por parte del consumidor español. Si analizamos las consecuencias del valor de marca, esto es, la disposición a pagar un precio primado, así como la intención de compra, la marca de cerveza importada Coronita, también obtiene una mejor valoración. Estos resultados son ciertamente reveladores y apuntan a que el consumidor español valora mejor a la marca importada y por tanto, estaría dispuesto a pagar un precio mayor por ella y tiene una mayor intención de comprar la cerveza de la marca Coronita, que la marca nacional analizada.

Estos resultados sugieren una reflexión, dado que si tenemos en cuenta la bibliografía previa, sería previsible obtener una mejor valoración de la marca de cerveza nacional -Mahou- por tres motivos que han sido ya comentados. E primero, porque según la teoría previa, los nacionales valoran mejor las marcas y productos nacionales a los importados (Knight, 1999). En segundo lugar, porque los consumidores tienen a valorar mejor las marcas procedentes de países con un mayor nivel de desarrollo (Wang y Lamb, 1983). En tercer y último lugar, porque los consumidores tienen una mejor percepción y valoración de los productos y marcas procedentes de países culturalmente similares al suyo (Tongberg, 1972; Cordell, 1991). Los resultados obtenidos parecen indicar, que esa previsible peor valoración del producto y la marca mexicana, 
no han tenido lugar. Muy al contrario, la marca de cerveza mexicana tiene una mejor valoración de marca que la cerveza nacional.

Así, los resultados obtenidos, están en la línea de los resultados obtenidos por Amine (2008) quien demostraba que en relación a la comida y bebidas importadas, la valoración de estos productos no depende exclusivamente de la imagen del país de origen, sino de otras variables como los esfuerzos y actividades de marketing de las compañías. De esta manera, los resultados parecen indicar que la empresa mexicana Coronita ha sabido posicionarme adecuadamente en el mercado español, que ha llevado a cabo actividades de marketing favorables y positivas. Entendemos que los resultados obtenidos están también en la línea propuesta por Rao y Monroe (1989), dado que el consumidor español, ante una marca importada y una compra de poca implicación y riesgo percibido, emplearía atributos extrínsecos, especialmente la marca, para su valoración y su decisión de compra. Por ello, debemos concluir que el esfuerzo de marketing realizado por la empresa mexicana ha permitido posicionar la marca Coronita como una marca con valor y que el consumidor español así lo percibe. Entendemos que estos resultados muestran que Coronita ha sabido posicionarse adecuadamente en el mercado español, y quizá lo haya hecho en virtud de su personalidad de marca, o mediante las características organolépticas del producto - sabor suave, sabor a limón-, o bien mediante otras variables de marketing como el precio, posicionándose como una marca con personalidad, de elevada relación calidad-precio y dirigiéndose a un segmento muy concreto del mercado español.

\section{IMPLICACIONES Y FUTURAS LÍNEAS DE INVESTIGACION}

De nuestro estudio se derivan una serie de implicaciones. La primera de ellas está relacionada con el atributo del producto que más valoran los consumidores españoles -el sabor-. Los gestores y directores de marketing de las compañías cerveceras deberían tener en consideración este resultado para incidir especialmente en este atributo del producto, a la hora de diseñar y llevar a 
cabo las campañas de comunicación y publicitarias. En segundo lugar, la dirección de marketing de las empresas del sector cervecero debería hacer especial hincapié en aquellas dimensiones del valor de marca con peor valoración, esto es, la lealtad de marca y el valor de marca general. Especial relevancia tiene la variable lealtad de marca, dado que como señalan numerosos estudios (Yoo et al., 2000), es la dimensión con mayor peso e influencia en el valor de marca. Por ese motivo, serían deseables actuaciones de marketing encaminadas a la fidelización de los consumidores de cerveza.

Como principal limitación de esta investigación debemos señalar el tamaño muestral, lo que confiere un carácter de provisionalidad a las conclusiones aportadas, y entendemos que por ese motivo no es posible la generalización de los resultados. En este punto debemos destacar el objetivo exploratorio y de aproximación a una realidad como es sector de la cerveza y en concreto a la valoración de los consumidores españoles de dos marcas diferentes, y con una estrategia de makerting mix bien diferenciada en el mercado. Entendemos que son necesarios nuevos estudios para incluir otras marcas de cerveza presentes en el mercado español, y que no se han analizado en este estudio exploratorio, tanto nacionales como importadas. También podría plantearse un estudio más en profundidad que abarcara diferentes países europeos en los cuales estuvieran presentes ambas marcas. Por último, como futura línea de investigación, entendemos conveniente incorporar la variable o constructo país de origen, como un antecedente del valor de marca, dado su relevancia para el estudio del valor de marca en el sector alimenticio y de la bebida.

\section{BIBLIOGRAFIA}

Aaker, D.A. (1991). Managing Brand Equity Capitalizing on the Value of Brand Name. New York: The Free Press.

Aaker, D.A. (1996). Measuring Brand Equity Across Products and Markets. California Management Review, Vol. 38, No. 3, pp. 102-120.

Agarwall, M.K. y Rao, V.R. (1996). An Empirical Comparison of ConsumerBased Measures of Brand Equity. Marketing Letters, Vol. 7, No. 3, pp. 237-247. 
Amine, L. (2008). Country of origin, animosity and consumer response: Marketing implications of anti-Americanism and Francophobia. International Business Review, Vol. 17, No. 4, pp. 402-422.

Arvidsson, A. (2006). Brand Value. Journal of Brand Management, Vol. 13, No. 3, pp. 188-192.

Atilgan, E., Aksoy, S. y Akinci, S. (2005). Determinants of the Brand Equity: A Verification Approach in the Beverage Industry in Turkey. Marketing Intelligence and Planning, Vol. 23, No. 2/3, pp. 237-248.

Bilkey, W. (1993). Foreword, in Product-Country Images: Impact and Role in International Marketing. Nicolas Papadopoulos and Louise Heslop, eds., New York: International Business Press.

Bilkey, W. y Nes, E. (1982). Country of Origin Effects on Product Evaluations. Journal of International Business Studies, Vol. 8, Spring/Summer, pp. 89-99.

Burke, W. y Schoeffler, S. (1980). Brand Awareness as a Tool for Profitability. The Strategic Planning Institute, Boston: Cahner Publishing Company, 1980.

Cobb-Walgren, C.J., Ruble, C.A. y Donthu, N. (1995). Brand Equity, Brand Preference and Purchase Intent. Journal of Advertising, Vol. 24, No. 3, pp. 25-40.

Cordell, V. (1991). Competitive context and price as moderators of country of origin preferences. Journal of the Academy of Marketing Science, Vol. 19, spring, pp. 123-128.

Chen, K.J. (2001). Using Free Association to Examine the Relationships between the Characteristics of Brand Associations and Brand Equity. Journal of Product and Brand Management, Vol. 10, No. 7, pp. 439-451.

Dean, D.H. (2004). Evaluating Potential Brand Associations through Conjoint Analysis and Market Simulation. Journal of Product and Brand Management, Vol. 13, No. 7, pp. 506-513.

Dekhili, S. y d'Hauteville, F. (2009). Effect of the region of origin on the perceived quality of olive oil: An experimental approach using a control group. Food Quality and Preference, Vol. 20, No. 7, pp. 525-532. 
Erdem, T., Swait, J. y Louviere, J. (2002). The impact of Brand Credibility on Consumer Price Sensitivity. International Journal of Research in Marketing, Vol. 19, pp. 1-19.

Farquhar, P. (1989). Managing Brand Equity. Marketing Research, Vol. 48, pp. 24-33.

Grover, R. y Srinivasan, V. (1992). Evaluating the Multiple Effects of Retail Promotions on Brand Loyal and Brand Switching Segments. Journal of Marketing Research, Vol. 29, pp. 76-89.

Jung, J. y Sung, E-Y. (2008). Consumer-Based Brand Equity: Comparison among Americans and South Koreans in the USA and South Koreans in Korea. Journal of Fashion Marketing and Management, Vol. 12, 1, pp. 24-35.

Keller, K. L (1993).Conceptualizing, Measuring and Managing Customer-Based Brand Equity. Journal of Marketing, Vol. 57, pp. 1- 22.

Keller, K.L. (2007). Strategic Brand Management: Building, Measuring and Managing Brand Equity, $3^{\text {rd }}$ Ed. New York: Prentice Hall.

Kim, K.H., Kim, K.S., Kim, D.Y., Kim, J.H. y Kang, S.H. (2008). Brand Equity in Hospital Marketing. Journal of Business Research, Vol. 61, pp. 75-82.

Knight, G.A. (1999). Consumer preferences for foreign and domestic products. Journal of Consumer Marketing, Vol. 16, 2, pp. 151-162

Lassar, W., Mittal, B. y Sharma, A. (1995). Measuring Customer-based Brand Equity. Journal of Consumer Marketing, Vol. 12, 4, pp. 11-19.

Myers, C.A. (2003). Managing brand equity: a look at the impact of attributes. Journal of Product and Brand Management, Vol. 12, No. 1, pp. 39-51.

Nedungadi, P. (1990). Recall and consumer consideration sets: influencing choice without altering brand evaluations. Journal of Consumer Research, Vol. 17, No. 3, pp. 263-276.

Netemeyer, R.G., Krishnan, B., Pullig, C., Wang, G., Yaggi, M., Dean, D., Ricks, J. y Wirth, F. (2004). Developing and Validating Measures of Facets of Customer-Based Brand Equity. Journal of Business Research, Vol. 57, pp. 209-224. 
Pappu, R., Quester, P.G. y Cooksey, R.W. (2005). Consumer-based Brand Equity: Improving the Measurement. Empirical Evidence. Journal of Product and Brand Management, Vol. 14, No. 3, pp. 143-154.

Pappu, R., Quester, P.G. y Cooksey, R.W. (2006). Consumer-based Brand Equity and Country-of-origin Relationships. European Journal of Marketing, Vol. 40, No. 5/6, pp. 696-717.

Pappu, R., Quester, P.G. y Cooksey, R.W. (2007).Country Image and Consumer-Based Brand Equity: Relationships and Implications for International Marketing. Journal of International Business Studies, Vol. 38, pp. 726-745.

Rao, A. y Monroe, K. (1989). The effect of Price, Brand name and store name on buyers' perceptions of product quality: An integrative review. Journal of Marketing Research, August, pp. 351-357.

Rossiter, J.R. y Percy, L. (1987). Advertising and Promotion Management. New York, NY: McGraw-Hill.

Verbeke, W. y Ward, R. (2006). Consumer interest in information cues denoting quality, traceability and origin: An application of ordered probit models to beef labels. Food Quality and Preference, Vol. 17, No. 6, pp. 453-467.

Wang, C. y Lamb, C. (1983). The impact of selected environmental forces upon consumers' willingness to buy foreign products. Journal of the Academy of Marketing Science, Vol. 11, pp. $71-84$.

Wilson, C. (1981). A procedure for the Analysis of Consumer Decision Making. Journal of Advertising Research, Vol. 21, No. 2, pp. 31-38.

Yoo, B., Donthu, N. y Lee, S. (2000). An examination of Selected Marketing Mix Elements and Brand Equity. Academy of Marketing Science, Vol. 28, No. 2, pp. $195-211$

Yoo, B. y Donthu, N. (2001). Developing and Validating a Multidimensional Consumer-based Brand Equity Scale. Journal of Business Research, Vol. 52, pp. 1-14.

Yoo, B. y Donthu, N. (2002). Testing Cross-Cultural Invariance of Brand Equity Creation Process. Journal of Product and Brand Management, Vol. 11, No. 6, pp. 195-211. 
Zeithaml, V.A. (1988), Consumer Perceptions of Price, Quality and Value: A Means-end Model and Synthesis of Evidence. Journal of Marketing, Vol. 52, No. 3, pp. 2-22. 